\title{
Kinetic Energy Harvesting from Human Hand Movement by Mounting micro Electromagnetic Generator
}

\author{
F. R.Pathan ${ }^{*}$ \\ Department of Electrical \& Electronic Engineering, Kaunas University of Technology-KTU, Lithuania. \\ Faculty of Sciences and Engineering, East West University, Bangladesh. \\ Department of Electrical Engineering, N.E.D University of Engineering \& Technology, Pakistan.
}

\begin{abstract}
A comprehensive review of design and experimentation is presented in this research paper on sustainable renewable energy scavenging from Human body movement using Micro electromagnetic kinetic energy harvester to powering wearable, portable electronics, implantable medical devices etc. The body location which is chosen as the harvester is human hand between elbow and shoulder. Human body harvest energy in two ways i,e, mechanical energy and thermal energy. Mechanical energy is of two kinds one is static energy and the other one is kinetic energy. Due to motion or displacement or enforcement excitation the kinetic energy is extracted. The electric charges which remains imbalance on the surface or within a material is static energy. Thermal energy is extracted from the dissipation of heat from human body. Human body parts and organs generate energy through two types of activities are voluntary and involuntary. The energy which are produced by voluntary activities are high as people intentionally does work by body motion, walk, run. The generated energy by involuntary organs like heart, breathing, artery are smaller compare to voluntary energy harvesting. One process of energy harvesting is by use of micro electromagnetic generator, flexible and stretchable piezoelectric, triboelectric, electromagnetic induction, PVDF cantilever mounting on human body. The harvester prototype is cylindrical magnet L40xD10 mm size which is mounted on human hand for energy harvesting. While in movement of hand the produced wave forms by magnetic generator are measured and recorded for calculation. Analyzing the received data it has been found that the generated power by micro electromagnetic vibration generator from movement of human hand are $319 \mathrm{RMS} \mu \mathrm{W}$ and $2.48 \mathrm{RMS} \mathrm{mV}$ with a frequency of $0.25 \mathrm{~Hz}$ and power density of about $2.48 \mu \mathrm{W} / \mathrm{cm}^{3}$.
\end{abstract}

\section{Introduction}

In this research innovation pursued Kinetic energy harvesting by a non- resonant micro electromagnetic generator based on free movement of human hand. Now most of the cases energy is stored in chemical battery with limited life span and which needs to recharge in some period. The battery is replaceable after limited time period which is costly and make lot of hazards. Human body motion is a big source of energy scavenging which is innovated by scientists as a significant development[1]. The majority of the systems are able to convert high frequency - low amplitude vibrations in to electrical energy [2]. A cylindrical prototype is used for this research purpose. While construction of prototype a magnet is used, a cylinder type tube covered by electrical coil winding 500 turn and closed two sides of cylinder by hard paper made stoppers. Due to the movement of the cylinder, the magnet which is placed inside moves freely from one side of cylinder to another side. In movement of the magnet inside the tube collides with the cylinder and the stoppers[3]. The free movement of magnet an electromagnetic induction is produced and power is generated in the covered electrical coil. So, through this technology power harvesting is received from electromagnetic generator.

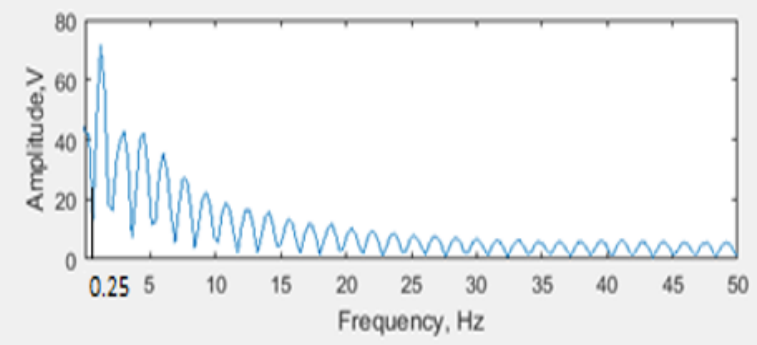

Fig.1. Low frequency and high amplitude signal.

A low frequency and high amplitude is produced in this free movement of magnetic generator [4] and creates a behavior of non resonant energy scavenging. It is proved of increase in output power due to faster movement of human hand and also increases the amplitude and frequency. Indeed, low frequency and large amplitude vibration is produced due to human every day

Corresponding author: frpathan@yahoo.com 
movement activities in walking and free motion of hand. Scientists applied different techniques to emphasis on such low frequency vibration by developing lot of kinetic energy harvesters [5].

\section{System configuration and fabrication}

The Micro Electromagnetic generator is made based on movement of human hand while walking. It is manufactured by hard paper as a tube cylinder type. The thickness of the paper is $0.2 \mathrm{~mm}$, inner diameter is 0.25 $\mathrm{mm}$, total length is $40 \mathrm{~mm}$ and diameter with $12 \mathrm{~mm}$. A strong NdFeB permanent magnet is placed inside the tube by closing two sides with glued of the tube consists of thin paper of stopper which prevents magnet to come out of the cylinder in oscillation. In hand motion the magnet moves inside the cylinder freely without any sticking with the tube or end cover stopper from free oscillation of the magnet[6].

While oscillation of magnet the two sides closing paper washers allow air flow in and out of the cylinder. In Figure. 2 it is shown that the shape of the tube consists of diameter $12 \mathrm{~mm}$ and length $40 \mathrm{~mm}$. The dimension of the magnet length is $30 \mathrm{~mm}$ and diameter is $10 \mathrm{~mm}$. To make an electrical coil an enameled copper wire of $0.2 \mathrm{~mm}$ is winding up outside the paper tube surface and secured in position by the tube flanges with 500 turns by manually copper coil winding.

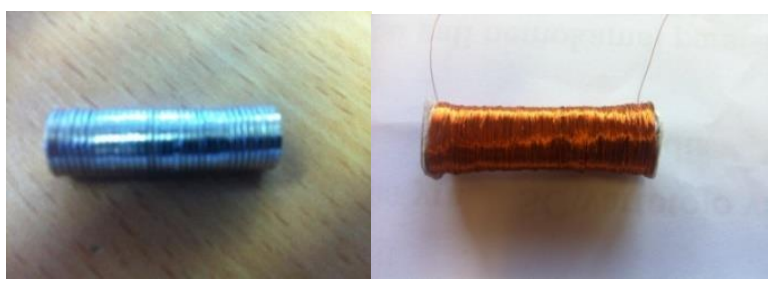

Fig.2. Micro electromagnetic generator and the Magnet used inside.

Multiple layer windings cover the coil consists of many horizontal turns and vertically one coil cover another coil while winding the coils on the surface of the cylinder. Measuring the resistance of the coil by multimeter found $40.3 \Omega$. The fabricated Micro electromagnetic generator is D12xL40 mm.

\section{Input vibration from human hand motion in walking}

Human body motion provides significant amount of vibration. In movement of human body along with Micro electromagnetic Generator acts the role for the performance of input vibration and output amplitude and frequency. Due to input amplitude and frequency power is increased. The vibration of human hand motion is multidirectional and in nature very much complicated. Due to such nature it effects on performance of magnet and cylinder oscillation and energy harvesting capacity. The reason for selecting the position between elbow and shoulder is that this part of hand makes more movement compare to other parts of body[7]. Also this is convenient to operate the system in powering up some low power devices. The micro electromagnetic generator is placed in between shoulder and elbow of hand in view of maintaining the scavenger cylinder tube with same direction axis of the human body movement which is shown in fig.7. Hand is found the most frequent moving part of human body from where significant amount of power can be extracted. While walking along with the movement of leg human also has to make motion of their hand. Frequency or stride rate and stride crossing length is being act on human walking gate speed [8]. In walking arm swing act along with leg and the input vibration depends on arm swing for harvesting energy from the harvester which is mounted in hand. In research it is found that the frequency ratio between the arm and leg movement at a walking speed in less than $0.8 \mathrm{~m} / \mathrm{s}$ is $2: 1$, while it is $1: 1$ and the arm moves in-phase with the leg at a speed higher than $8 \mathrm{~m} / \mathrm{s}$,[9].

\section{Power signal}

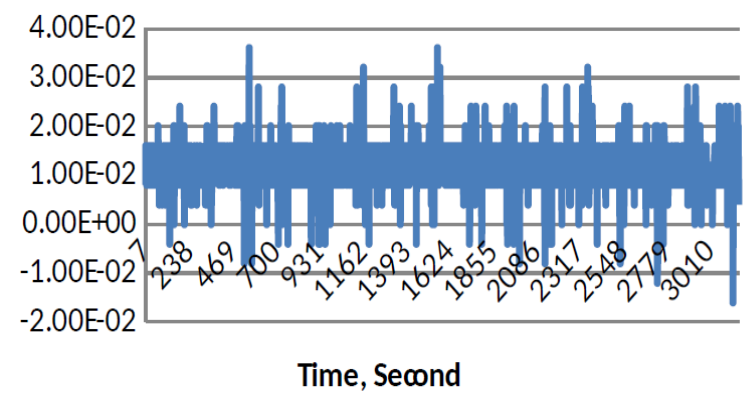

Fig.3. Voltage waveform of cylindrical-magnet prototype while attached to the hand.

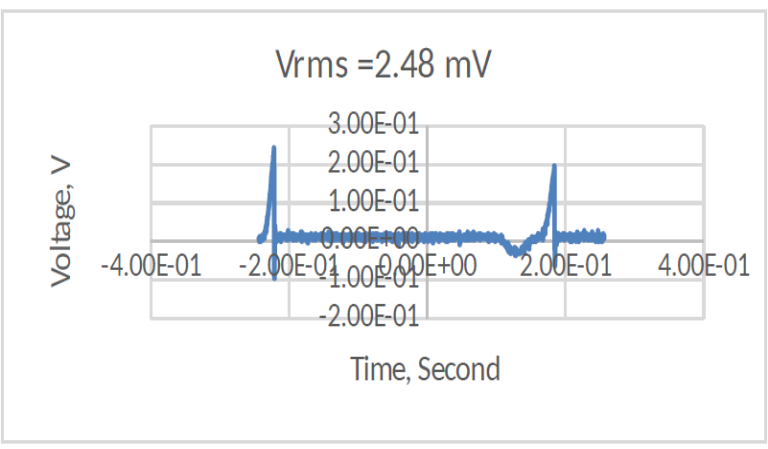

Fig.4. Measured voltage wave form of cylindrical-magnet prototype attached in hand.

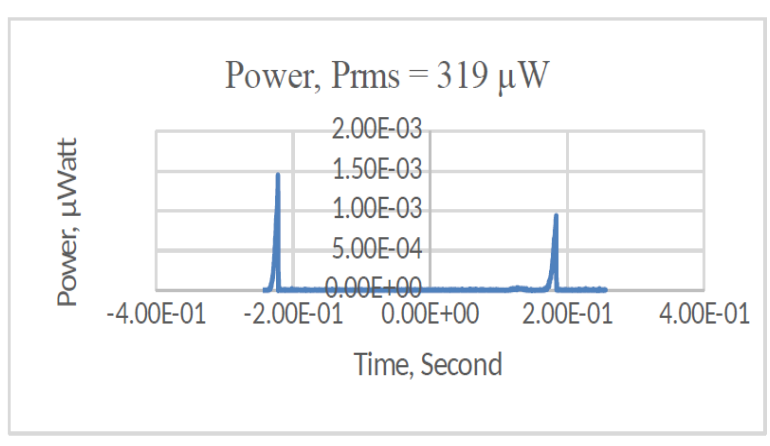

Fig.5. Measured Power signal of the cylindrical-magnet prototype fixed to the hand. 


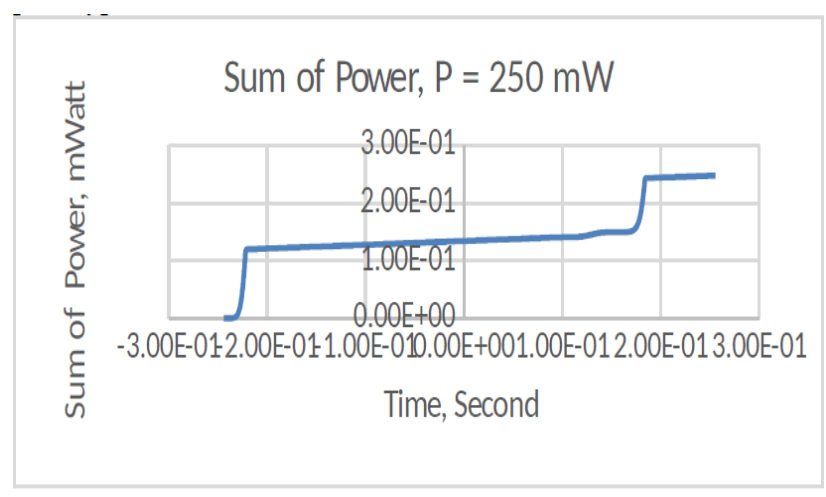

Fig.6. Sum of Power signal of the cylindrical-magnet prototype in attached to the hand.

The increasing of the walking speed increases the amplitude of arm swing and output power harvesting[10]. To get increase volume of power from the hand fixed harvester depends on increase of frequency and amplitude increase at hand.

\section{Principle of power generation by micro electromagnetic generator}

Human being move their hands in front and back while they walk in gate cycle. Due to this free motion of hand the magnet inside the cylinder which is being mounted on human hand also shake front and back. For such magnet shaking inside the cylinder of micro electromagnetic generator a positive and negative voltage develops in two sides of the coil which is wounded outside on the surface of the cylinder. Magnet has fixed magnetic flux. While moving magnet in the coil generates voltage due to moving magnetic field penetrating coil windings. So, the electricity is produced due to the total energy of the motion of the magnet and the magnetic field within a wire of copper coil produce the electrons to move in the wire.

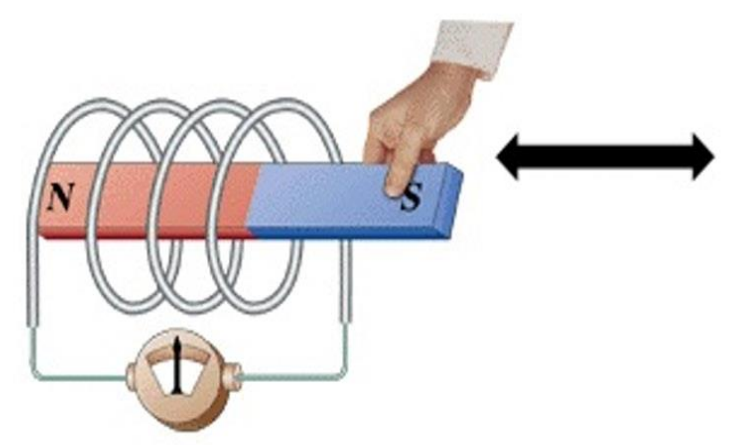

Fig.7. Electromagnetic Induction in Power generation.

So, the electric current is produced due to the total energy of the motion of the magnet and the magnetic field within a wire of copper coil produce the electrons to move in the wire. Two terminals of copper coil are connected to capacitor or rechargeable battery. This generated power is extracted from the coil to super capacitor or rechargeable battery and preserve for charge up low power devices.

Inside a copper coil if permanent magnet is moved or shacked it would produce a changing magnetic field. So, in the copper coil a voltage would be induced due to the changing magnetic field.

\section{Application of micro electromagnetic generator}

Micro electromagnetic generator can be used in such a place or environment where conventional battery can't function like in sea wave, river water, canal, rain to measure the different parameters of water for example temperature of water, pollution of water, depth of the sea or river, identifying the location of drowned water vessel by generating energy from micro electromagnetic generator attaching it with a ship or water operating vessel. Conventional chemical batteries get leaked out, damage if use battery in water. Micro electromagnetic generator can be used in water putting it inside a water proof pot. Fixing micro electromagnetic generator with outside surface body of ship, launch, steamer, speed boat can harvest energy by the vibration of the wave that strikes the water vessel.

This is suitable for the use in measuring temperature, humidity, industrial wireless sensing, surplus heat energy harvesting, remote sensors and radio power, predictive maintenance, automation, RFID, real time locator, transport tracking, cars sensors, pursuer, intrusion detection, emergency medical response monitoring, pacemaker, defibrillator, bearing vibration, air pollution monitoring, cargo monitoring and tracking, wireless bridge monitoring, active tags for real time tracking and logistics, oil refinery monitoring, medical implantations, medical remote sensing, body area network, tire pressure monitoring (TPM), pipeline corrosion monitoring and distributed wireless multi-sensor.

\section{Maintenance reduction and access in harsh environments}

Micro Electromagnetic generator is absolutely a device which does not need any maintenance. This is completely cost free maintenance generator. Due to such quality of maintenance free in terms of cost and technicality, micro electromagnetic generators are in deployment of thousands of sensors and sensors with MEG are implementing in backward locations. So, this technology is the most suitable and convenient in sensor nodes without battery since they possess infinite life time without any trouble. Micro Electromagnetic generator also functions in adverse and harsh environment. Even over $80^{\circ} \mathrm{C}$ temperature they function efficiently for long years. Whereas batteries are no match with micro electromagnetic generator at such a high temperature environment. Implementing this technology of energy harvesting in every year millions of chemical batteries can be avoided to throw in the environment. 


\section{The results of experiment}

The time of carrying this experiment measured and recorded the voltage wave forms which were generated by the free motion of the magnet inside the prototype fixed on hand in between shoulder and elbow. From this experiment received wave forms are shown in fig.4. The voltage and power generation at low and high frequencies depend on magnetic shape, its friction and magnetic characteristics. Chosen a man of 52 years of age, $168 \mathrm{~cm}$ in height and $60 \mathrm{Kg}$ of weight for conducting this experiment. An amount of power has been extracted from the hand by free motion of fixing micro electromagnetic generator. From this cylindrical prototype harvester RMS power $319 \mu \mathrm{W}$ has been harvested during walking which is shown in Fig.5. Lower friction resulted in higher magnet/tube relative motion from such low frequency motion and consequently higher induced voltage. Harvesting energy from hand increases due to increase of hand movement and increase of frequency and amplitude input.

\section{Final Remarks}

Energy harvesting by using micro electromagnetic generator on human hand in free movement of hand gate has been invented in this research work. In fabrication of micro electromagnetic generator is used a magnet inside a cylindrical tube wounded 6 .by coil of 500 turns where the magnet can freely move inside cylinder in walk of human being. While the free motion of human hand an oscillation occur of hand in walk and create an impact between cylinder and magnet and end stopper of the micro electromagnet generator in which amplitude and frequency appear in the signal. Power harvesting by micro electromagnetic generator in a non-resonance nature in low frequency vibration and high amplitude. The research shows that it generates low frequency and large amplitude from the output power. In this research is received 319 $\mu \mathrm{W}$ RMS power and $2.48 \mathrm{RMS} \mathrm{mV}$ at $0.25 \mathrm{~Hz}$ frequency with power density of $2.48 \mu \mathrm{W} / \mathrm{cm}^{\wedge} 3$. The generation of power will be higher due to higher input of frequency and amplitude and the output will be more. So, applying higher input amplitude generates large output amplitude vibration and more output power. In free motion of the magnet inside the tube must synchronize with the shaking force exerts on stopper.

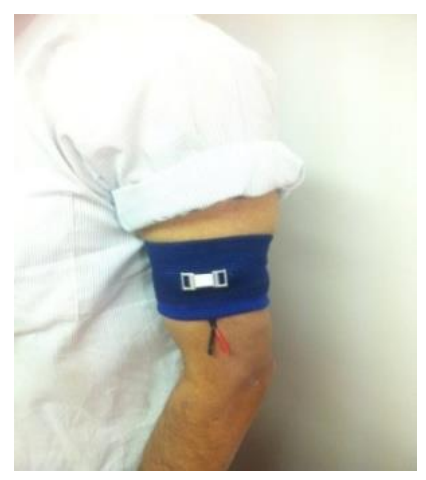

Fig.8. Harvester Electromagnetic generator placed on hand.

\section{Acknowledgement}

This work is guided and supported by my Ph.D Supervisor Prof Dr. Vytautas Markevicius, Professor, Department of Electrical \& Electronic Engineering, Kaunas University of Technology-KTU, Lithuania.

\section{References}

1. Ross Kerley, Xiucheng Huang and Dong Sam Ha et al.( 2016 ) Energy Harvesting from the Human Body and Powering up Implant Devices.

2. Iannacci J, Serra E, Criscienzo R Di, Sordo G, Gottardi M, et al. (2014) Multi-modal vibration based MEMS energy harvesters for low power wireless functional nodes. MicrosystTechnol20: 627-640.

3. Ahmed Harouna,b,*, Ichiro Yamada c, Shin'ichiWarisawac et al.( 2015 ) Micro electromagnetic vibration energy harvester based on free/impact motion for low frequency-large amplitude operation.

4. T. Von Büren, P.D.Mitcheson, T.C.Green, E.M.Yeatman, A.S. Holmes, G. Tröster, Optimization of inertial micro power generators for human walking motion, Sensors (Peterborough, NH) 6 (1) (2006).

5. Miah A, Halim Park, Jae Y (2014) A non-resonant, frequency up-converted electromagnetic energy harvester from human-body-induced vibration for hand- held smart system applications. J. Applied Physics 115: 094901.

6. Ahmed Harouna,b, Ichiro Yamada c, Shin'ichi Warisawac et al.( 2015 ) Micro electromagnetic vibration energy harvester based on free/impact motion for low frequency-large amplitude operation.

7. P. Miao, P.D. Mitcheson, A.S. Holmes, E.M. Yeatman, T.C. Green, B.H. Stark, MEMS inertial power generators for biomedical applications, Micro syst. Technol. 12(2006) 1079-1083.

8. T. Von Büren, P.D. Mitcheson, T.C. Green, E.M.Yeatman A.S. Holmes, G.Tröster, Optimization of inertial micro power generators for human walking motion, IEEE Sens. 6 (2006) 28-38.

9. Ahmed Haroun, Ichiro Yamada, Shin'ichi Warisawa,etal (2016), Investigation of Kinetic Energy Harvesting from Human Body Motion Activities using Free/ Impact Based Micro Electromagnetic Generator.

10. S.M. Jung, K.S. Yun, Energy-harvesting device with mechanical frequency-up conversion mechanism for increase power efficiency and wideband operation, Appl. Phys. Lett. 96 (2010), 11906. 